\title{
A SURVEY OF MICRODOSIMETRIC QUANTITIES AND CONCEPTS
}

\author{
A.M. KELLERER \\ Institut für medizinische Strahlenkunde der Universität Würzburg. Versbacherstrasse 5, D-8700 Würzburg. Fed. Rep. of Germanv
}

Received 4 July 1984

Basic concepts of microdosimetry are given. The need for and the definitions of basic quantities are presented, along with remarks concerning experimental methods, computational techniques and recent developments.

\section{Introduction}

Ionizing radiation transfers energy to the exposed materials in discrete random events. Microdosimetry is concerned with the resultant microscopic patterns of energy that determine the biological effectiveness of different types of ionizing radiation $[1,2]$. It has been applied predominantly to radiobiological problems, but has recently also been utilized in the study of radiation damage to electronic components, such as the generation of soft errors in computer memories [3,4]. Microdosimetry has not, up to now, been applied to the analysis of material damage in solids by very large doses of ionizing radiation; and, especially, the possible role of atomic displacements has not been treated. In its present form microdosimetry has therefore restricted applicability to the problems of radiation damage in cryomicroscopy. A synopsis of the essential quantities is nevertheless useful, if only to facilitate the construction of concepts that are more closely linked to the processes of solid state damage.

\section{Basic concepts}

Absorbed dose, $D$, is defined as a statistical expectation value which loses its meaning when applied to microscopic regions where the statistical fluctuations of imparted energy can be large. Classical target theory [5,6] utilized a highly sim- plified description of the stochastic nature of energy deposition in terms of the Poisson distribution of the number of hits which were alternately thought to be events of point heat [7] or single ionizations [8]. It was soon realized that the statistics of energy deposition is far too complex to be described as a pure Poisson process. The additional notion introduced to quantify the distribution of energy on a microscopic scale has been the mean rate of energy loss of a charged particle; this parameter has been termed mean ionization density, linear energy transfer (LET), or electronic collision stopping power (see fig. 1).

However, stopping power itself is merely a statistical expectation value. The energy actually imparted by an ionizing particle of stopping power $L$ along a track segment $\Delta x$ can deviate substantially from its expected value, $L \Delta x$ (see fig. 2). Energyloss straggling and energy transport by $\delta$-rays are the main factors responsible for the differences, and their combined influence is greatest for regions that are considerably smaller than $1 \mu \mathrm{m}$. For heavy ions there are certain combinations of site sizes and energies of the particles for which the LET concept remains applicable. For electrons there are no such conditions; application of the LET concept never permits the precise prediction of energy deposition in a microscopic site [10].

Microdosimetry originated when Rossi and coworkers realized the inherent impossibility to measure LET distributions. They concluded that actual cnergy concentrations in microscopic regions 


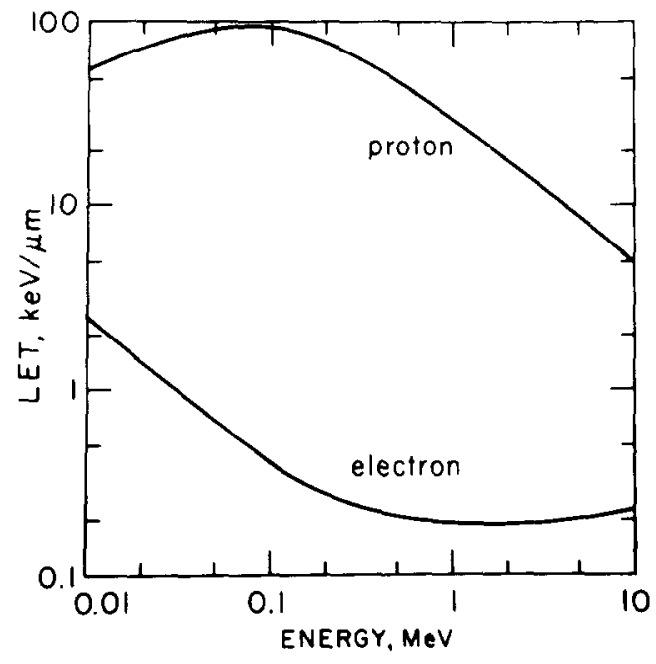

Fig. 1. Collision stopping power, also termed linear energy transfer, for electrons and protons in tissue (or water) as function of their energy [9].

are more relevant to radiation effects than the theoretical values computed on the basis of LET distributions [11,12]. Based on this recognition a conceptional framework was then constructed to deal with the actual random variables rather than their statistical mean values, and experimental methods were developed to simulate the distributions of the microdosimetric variables in tissue in spherical proportional counters that are filled with tissue-equivalent gas and that have tissue-equivalent walls (see refs. $[1,2]$ ).

\section{Definition of basic quantities}

The essential notion in microdosimetry is that of specific energy which is the random variable corresponding to absorbed dose. Although reference is made most frequently to this variable, it is practical to introduce a set of related quantities that are given in the subsequent list. For rigorous definitions, see refs. $[1,2]$.

Energy imparted: The energy imparted, $\epsilon$, by ionizing radiation to the matter in a volume is the difference between the energy of ionizing radiation entering the volume and that emerging from it. Transformations of rest mass into energy of ionizing radiation within the volume are added to the resulting term.

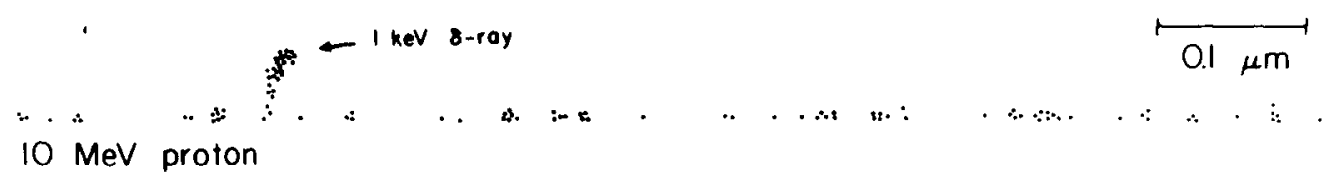

$500 \mathrm{keV}$ proton

I MeV electron

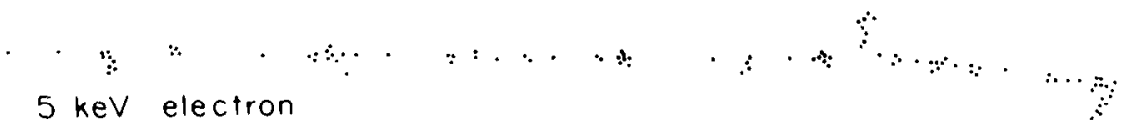

Fig. 2. Schematic diagram of track segments of charged particles in tissue (or water). Each dot stands for an energy transfer of $30 \mathrm{eV}$ on the average. The lateral extension of the tracks is exaggerated in order to visualize all energy transfers [9]. 
Specific energy: The specific energy, $z$, in a mass $m$ is the quotient of $\epsilon$ by $m$. The unit of specific energy is $\mathbf{J ~ k g}^{-1}$; its special name is gray (Gy). The former special unit of specific energy, as of absorbed dose, has been the $\mathrm{rad}(=0.01 \mathrm{~Gy})$.

$z$ is a random variable. It is, therefore, useful to consider its probability distribution. Specific energy in a volume may be due to one or more energy deposition events. An event is energy deposition by an ionizing particle and/or its secondaries. The distribution function, $F_{1}(z)$, of the specific energy deposited in a volume is the conditional probability for a specific energy not larger than $z$, if one event has occurred. The probability density, $f_{1}(z)$, is the derivative of $F_{1}(z)$ with respect to $z$ :

$f_{1}(z)=\frac{\mathrm{d} F_{1}(z)}{\mathrm{d} z}$.

The value of the distribution function, $F(z)$, of $z$ at a specified absorbed dose is equal to the probability that the specific energy is not larger than $z$. The probability density, $f(z)$, is the derivative of $F(z)$ with respect to $z$ :

$f(z)=\frac{\mathrm{d} F(z)}{\mathrm{d} z}$.

In contrast to the single event distribution, $f(z)$ includes a discrete component (a Dirac $\delta$-function) at $z=0$ for the probability of no energy deposition. The dose-dependent distributions, $f(z)$, can be computed from the single event distributions, $f_{1}(z)$. Only the latter need, therefore, be measured (see later paragraph).

The absorbed dose, $D$, is equal to the mean specific energy:

$D=\bar{z}=\int_{0}^{\infty} z f(z) \mathrm{d} z$.

Absorbed dose (unit: $\mathrm{J} / \mathrm{kg}=\mathrm{Gy}$ ) has replaced the quantity exposure (unit: $\mathrm{C} / \mathrm{kg}$; former unit $R=$ $2.58 \times 10^{-4} \mathrm{C} / \mathrm{kg}$ ) which had been defined in terms of charge liberated in air and which had applied to photons only.

Table 1 illustrates the magnitude of doses $D$ relevant to radiation biology, on the one hand, and the far larger doses in electron microscopy, on the other hand.

Lineal energy: The lineal energy, $y$, is the quo-
Table 1

Comparison of orders of magnitude of absorbed doses and of the corresponding fluences of $50 \mathrm{keV}$ electrons; to indicate the extent of the fluctuations of energy imparted, the quantity $d$ is given in the last column; it is equal to the side length of that cube for which the standard deviation of energy imparted at the specified dose is $50 \%$

\begin{tabular}{llll}
\hline & $\begin{array}{l}\text { Absorbed } \\
\text { dose } \\
(\mathrm{Gy})\end{array}$ & $\begin{array}{l}\text { Fluence } \\
\left(\mathrm{nm}^{-2}\right)\end{array}$ & $\begin{array}{l}d \\
(\mathrm{~nm})\end{array}$ \\
\hline $\begin{array}{l}\text { Inactivation of } \\
\text { mammalian cells }\end{array}$ & 5 & $4.5 \times 10^{-5}$ & 1100 \\
$\begin{array}{l}\text { Inactivation of } \\
\text { viruses } \\
\text { "Low dose" } \\
\text { in electron } \\
\text { microscopy }\end{array}$ & $10^{3}$ & 0.009 & 110 \\
$\begin{array}{l}\text { "High dose" } \\
\text { in electron } \\
\text { microscopy }\end{array}$ & $1.1 \times 10^{5}$ & 1 & 13 \\
\hline
\end{tabular}

tient of the energy imparted, $\epsilon$, to the volume of interest and the mean chord length, $\bar{l}$, in that volume:

$y=\epsilon / \bar{l}$.

The mean chord length (for uniform isotropic randomness) of a convex volume is equal to 4 times its volume divided by its surface [13].

Lineal energy is related to energy increments produced by single events. The distributions of lineal energy are defined in analogy to the distributions of specific energy produced by single events. Since lineal energy is a random analogue of LET (collision stopping power) it is conventionally expressed in the same units, $\mathrm{keV} / \mu \mathrm{m}$. Measurements that aim at the determination of LET distributions determine, in actuality, the distribution of lineal energy. Due to energy-loss straggling and other stochastic factors these distributions can differ substantially from the distributions of LET.

\section{Need for microdosimetric quantities}

Microdosimetric quantities, such as specific energy, are required whenever one considers regions 
small enough that the relative fluctuations of energy deposition are substantial. As indicated in table 1, the relative fluctuations are largest for small volumes and small doses. At doses of a few Gy, which are of main interest in biological studies, the microdosimetric fluctuations are important for reference regions of fractions of a micrometer to several micrometers. At the always far higher doses to electron-microscopy samples the fluctuations are substantial only for regions of $10 \mathrm{~nm}$ or less.

A related point of view is the consideration of event frequencies. It is evident that microdosimetry is required whenever one deals with small or moderate event frequencies. Fig. 3 indicates those combinations of doses and site sizes for which the mean event frequency in the specified regions is below 1. Dose relations must be linear if the effects occur in regions where event frequencies are substantially below 1 . The number of affected domains is then proportional to dose; the energy imparted to the sites does not depend on ahsorbed dose but merely on radiation quality. Non-linear dependences on absorbed dose can occur only if the doses and the interaction distances are large enough to fall outside the shaded areas in fig. 3. At the high doses in electron microscopy cumulative effects are possible, even if the interaction distances between electronic disturbances are small. A plot analogous to fig. 3 but for atomic displacements would be very different; but basic arguments and concepts remain similar.

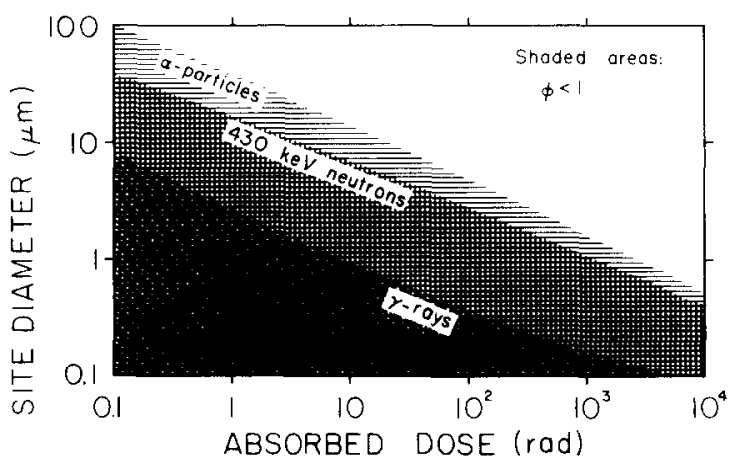

Fig. 3. The shaded areas indicate those combinations of site sizes and absorbed doses that correspond to mean event numbers less than unity.
The biological effects of ionizing radiation depend on the spatial and temporal correlation of energy within the nucleus of the cell. Enzymatic repair of DNA lesions tends to result in misrepair when lesions occur in close proximity. Energy imparted is therefore more effective if it occurs in dense clusters. The relative biological effectiveness of ionizing radiation increases up to a stopping power of about $100 \mathrm{keV} / \mu \mathrm{m}$; at even higher stopping powers, i.e. for heavy ions, the effectiveness decreases due to saturation. Io account for the increased effectiveness of densely ionizing radiation the quantity dose equivalent is utilized in radiation protection. It is equal to absorbed dose times a quality factor which is defined in terms of LET. To assess quality factors for unknown radiation fields one needs to determine the LET distributions. As stated above, the measurable quantity is lineal energy, $y$, rather than LET. In future, quality factors may, therefore, be linked to microdosimetric quantities.

The effects of ionizing radiation on simpler structures, such as viruses or enzymes, are due to single ionizations. The LET dependence is then reversed. Sparsely ionizing radiation has the highest effectiveness; densely ionizing radiation exhibits saturation and has less effect per unit absorbed dose.

For damage induced by ionizing radiation in solids there may be different dependences on ionization density or on the microdosimetric variables, whether one deals with cumulative or non-cumulative effects. In the latter case there may be saturation of damage in individual particle tracks or, at very high doses, saturation due to the superposition of independent particle tracks.

\section{Experimental methods}

Since its inception, microdosimetry has been largely concerned with problems of radiobiology and radiation protection. The emphasis has, accordingly, been on the analysis of patterns of energy deposition measured on a scale comparable to that of the nucleus of a mammalian cell or certain of its components. As it happens, measurements with proportional counters are restricted to 